\title{
Searches for new physics in dijet and multijet final states
}

\author{
Federico Preiato* on behalf of the CMS Collaboration \\ Sapienza Universita e INFN, Roma I (IT) \\ E-mail: federico.preiatodcern.ch
}

Searches for new physics in the dijet and multijet final states are presented from proton-proton collisions at $\sqrt{s}=13 \mathrm{TeV}$. The results shown include model-independent and model-specific searches using the dijet invariant mass spectrum and the dijet angular distributions, searches for black holes, quantum and microscopic, in multijet events, as well as searches for RPV SUSY in events with paired dijets. The most recent results obtained using data collected during the 2016 by the CMS experiment are presented.

The European Physical Society Conference on High Energy Physics

5-12 July

Venice, Italy

\footnotetext{
${ }^{*}$ Speaker.
} 


\section{Introduction}

The Standard Model of particle physics (SM) is the theory that summarizes our current understanding of particle physics and their interactions. From an experimental point-of-view, all the measurements performed in the last decades verified at a high precision level its predictions and no evidence of physics beyond the SM has been observed so far. Despite its experimental success, the SM is widely considered an incomplete theory for many reasons (e.g. it doesn't contain any viable dark matter particle).

Several alternative theories to the Standard Model have been elaborated to include the SM and to overtake such open issues in a more general theoretical framework. Many of these theories predict the existence of new particles that couple to quarks and/or gluons which could be produced at the LHC. These new particles could be therefore observed as bump in the dijet mass spectrum or as modification of some dijet event variable and they are the subject of the searches presented, performed by the CMS experiment [1].

\section{Dijet analysis [2]}

The dijet analysis is focused on the search for bump over the falling QCD spectrum. Two searches for dijet resonances are performed with the 2016 data. The first is a low-mass search for resonances with mass between 0.6 and $1.6 \mathrm{TeV}$ using dijet events corresponding to an integrated luminosity of $27 \mathrm{fb}^{-1}$. The events are reconstructed, selected, and recorded in a compact form by the high-level trigger (HLT) in a technique called data scouting. This technique allows to reduce significantly the trigger thresholds by significantly reducing the events size compared to the standard CMS data stream. The second is a high-mass search for resonances with mass above 1.6 $\mathrm{TeV}$ using dijet events that are reconstructed offline corresponding to an integrated luminosity of $36 \mathrm{fb}^{-1}$.

Figure 1 shows the dijet mass spectra, defined as the observed number of events in each bin divided by the integrated luminosity and the bin width, with predefined bins of width corresponding to the dijet mass resolution. The fits to the spectra are performed with the Equations 2.1 fitting the whole mass range in both cases.

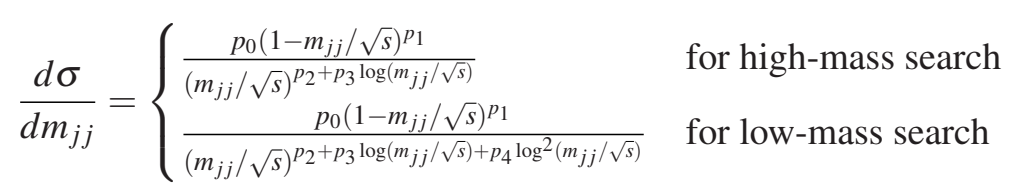

The dijet mass spectra, the background parameterization, and the dijet resonance shapes are used to set limits on the production of new particles decaying to parton pairs. Because of the dependence of the dijet resonance shape on the types of the two final-state partons (see Figure 2), separate limits are determined for each final state $(q q, q g$, and $g g)$ as shown in Figure 3. The dijet searches allow to put constraints on nine benchmark models, reported in Table 1.

\section{Boosted dijet analysis [3]}

This search considers a hypothetical resonance produced with sufficiently high transverse momentum that its decay products are merged into a single massive jet with two-prong substructure. 

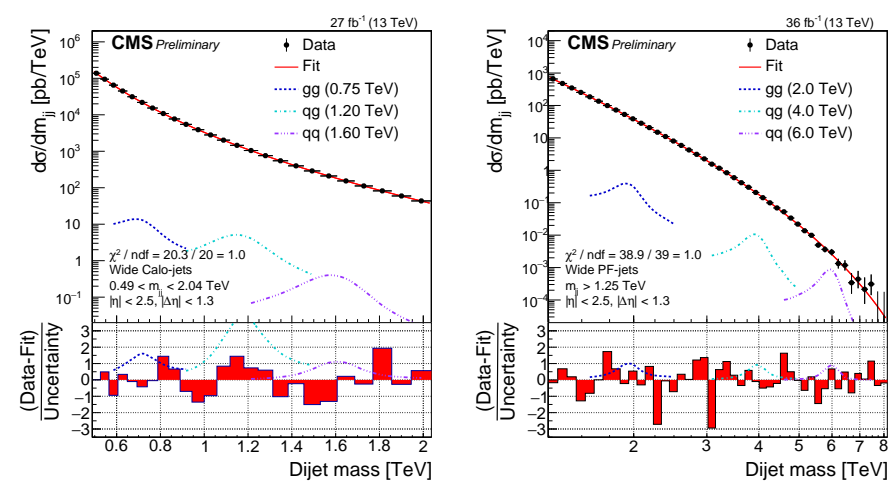

Figure 1: Dijet mass spectra (points) compared to a fitted parameterization of the background (solid curve) for the low-mass search (left) and the high-mass search (right) [2].

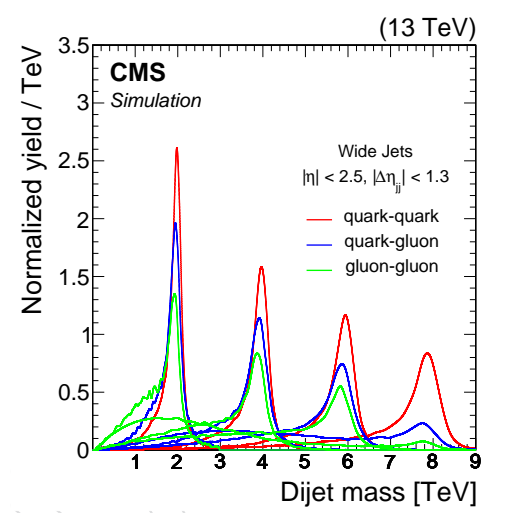

Figure 2: Signal shapes of narrow resonances with a mass of 2, 4, 6 and $8 \mathrm{TeV}$ in the high-mass search [2].

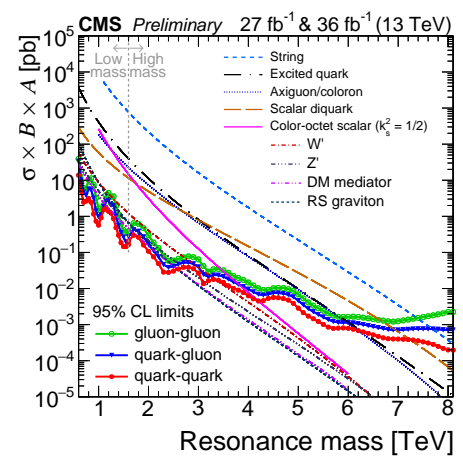

Figure 3: The observed 95\% CL upper limits on the product of the cross section, branching fraction and acceptance for the three possible final states. Limits are compared to predicted cross sections for the benchmark models considered in the analysis [2].

The approach is focused on the events where at least one high transverse momentum jet from initial-state radiation (ISR) is produced in association with a light resonance decaying into a $q \bar{q}$ pair (see Figure 4). The ISR requirement provides enough energy in the event to satisfy the trigger, either by the ISR jet or by the resonance itself. This strategy is complementary to the data scouting allowing to probe the region mass below $300 \mathrm{GeV}$.

The signal is identified as a peak over a smoothly falling background in the distribution of the invariant mass of the jet, using novel jet substructure techniques. In fact the massive jet is required to have the two-prong substructure expected from signal. The dominant background from QCD multijet production is estimate from data using control region created by inverting the substructure requirement. A binned maximum likelihood fit to the observed shape of the jet mass distribution is performed in five $p_{T}$ ranges whose boundaries are: 500, 600, 700, 800, 900 and $1000 \mathrm{GeV}$. The search for leptophobic vector (Z') resonance decaying to quark-antiquark pairs is performed using data collected by the CMS in 2016 and no significant excess have been observed. The results 


\begin{tabular}{lccccc}
\hline \hline \multirow{2}{*}{ Model } & \multicolumn{5}{c}{ Observed (expected) mass limit [TeV] } \\
& Final & $36 f b^{-1}$ & $12.9 \mathrm{fb}^{-1}$ & $2.4 \mathrm{fb}^{-1}$ & $20 \mathrm{fb}^{-1}$ \\
& State & $13 \mathrm{TeV}$ & $13 \mathrm{TeV}$ & $13 \mathrm{TeV}$ & $8 \mathrm{TeV}$ \\
\hline String & $q g$ & $7.7(7.7)$ & $7.4(7.4)$ & $7.0(6.9)$ & $5.0(4.9)$ \\
Scalar diquark & $q q$ & $7.2(7.4)$ & $6.9(6.8)$ & $6.0(6.1)$ & $4.7(4.4)$ \\
Axigluon/coloron & $q \bar{q}$ & $6.1(6.0)$ & $5.5(5.6)$ & $5.1(5.1)$ & $3.7(3.9)$ \\
Excited quark & $q g$ & $6.0(5.8)$ & $5.4(5.4)$ & $5.0(4.8)$ & $3.5(3.7)$ \\
Color-octet scalar $\left(k_{s}^{2}=1 / 2\right)$ & $g g$ & $3.4(3.6)$ & $3.0(3.3)$ & - & - \\
$W^{\prime}$ & $q \bar{q}$ & $3.3(3.6)$ & $2.7(3.1)$ & $2.6(2.3)$ & $2.2(2.2)$ \\
$Z^{\prime}$ & $q \bar{q}$ & $2.7(2.9)$ & $2.1(2.3)$ & - & $1.7(1.8)$ \\
RS Graviton & $q \bar{q}, g g$ & $1.7(2.1)$ & $1.9(1.8)$ & - & $1.6(1.3)$ \\
DM Mediator $\left(m_{\mathrm{DM}}=1 \mathrm{GeV}\right)$ & $q \bar{q}$ & $2.6(2.5)$ & $2.0(2.0)$ & - & - \\
\hline
\end{tabular}

Table 1: Mass limits at 95\% CL compared to previously published limits on narrow resonances from CMS. The listed models are excluded between $0.6 \mathrm{TeV}$ and the indicated mass limit by this analysis [2].

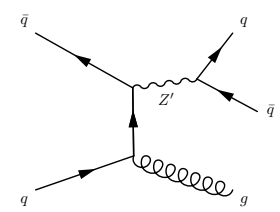

Figure 4: Production of $Z^{\prime}$ decaying into two jets in association with a jet from initial state radiation [3].

are therefore interpreted in terms of $95 \%$ confidence level (CL) upper limits on the resonance production cross section. An example of the jet mass distribution in the range 900-1000 GeV is shown in Figure 5 while in Figure 6 the upper limits as a function of the resonance mass are reported. These limits are compared to cross sections for a model of a leptophobic Z' resonance with a quark coupling $g_{q^{\prime}}$ of 0.17 and 0.08 .

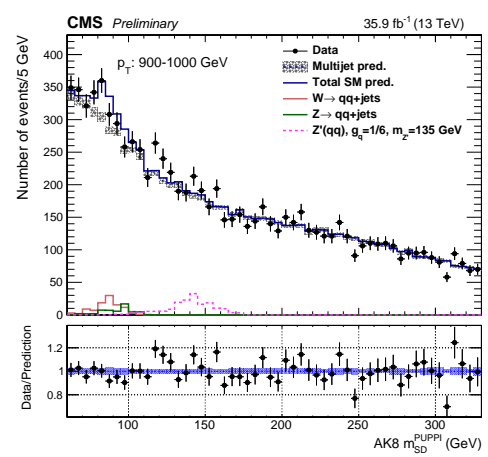

Figure 5: Jet mass distribution for the last $p_{T}$ category $(900-1000 \mathrm{GeV})$. Data (points) are in good agreement with the QCD background prediction (gray boxes), as also shown from the data/MC ratio reported on the bottom panel [3].

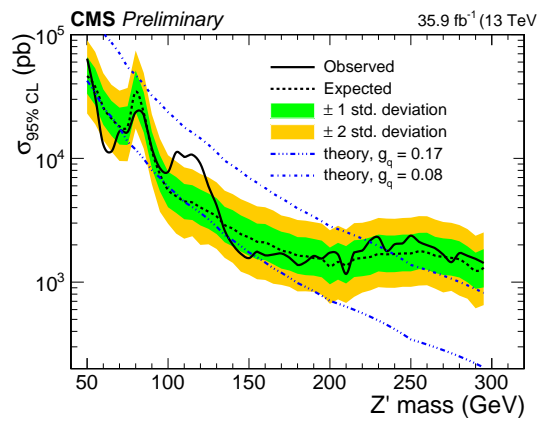

Figure 6: 95\% CL upper limits on the Z' production cross section compared to the theory with two different quark couplings [3].

\section{Pair-produced dijet analysis [4]}

The top quark superpartner (top squark or stop) is predicted by Supersymmetric (SUSY) models and it's required to be light. The pair production of stops decaying to light quarks via hadronic R-parity violating (RPV) is the process considered in this analysis (see Figure 7). For this purpose 
the search is focused on pair production of resonances decaying to pairs of light-flavor quarks in events where the resonant particles are boosted, resulting in a final state with two massive jets with substructure. This search uses data corresponding to an integrated luminosity of $2.7 \mathrm{fb}^{-1}$ recorded by CMS in 2015. The result from a search for paired boosted diquark resonances, using jet substructure techniques, is reported in Figure 8 which shows the comparison of the data in the signal region with the final total background prediction. No excess of events are observed in data and therefore upper limits at $95 \% \mathrm{CL}$ are set on the production of top squarks decaying to two light quarks in the framework of RPV supersymmetry. Top squarks with masses between 80 and 240 $\mathrm{GeV}$ are excluded.
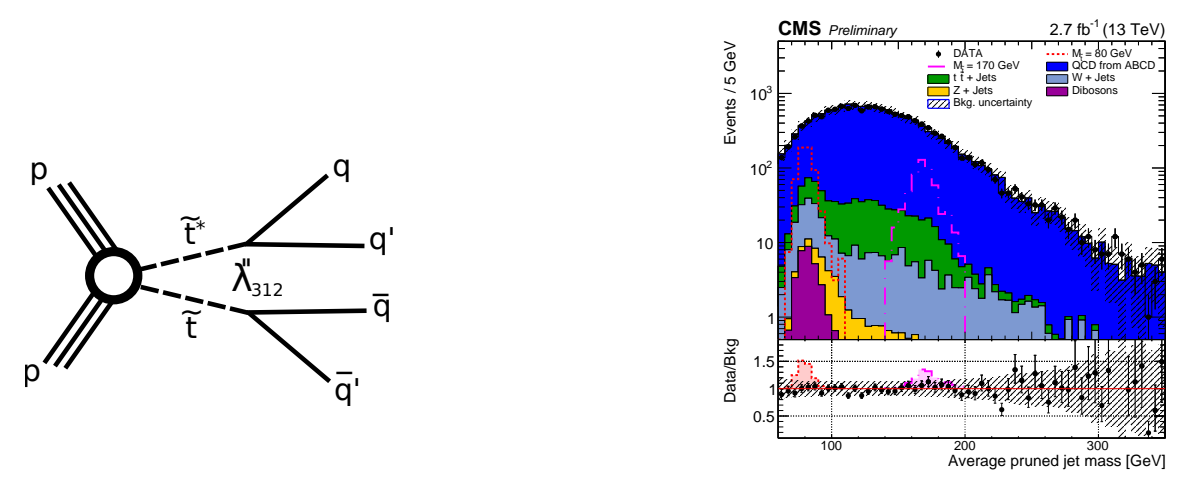

Figure 7: Direct pair production of stops decaying ${ }_{\text {Figure 8: Jet mass distribution shown for data (points) }}$ into two light quarks [4]. and the total background prediction. On the bottom, ratio between data and background prediction is reported [4].

\section{Dijet angular analysis [5]}

Wide resonance or non-resonant signatures can be probed exploiting the dijet angular distributions. This is typically expressed in terms of $\chi_{\text {dijet }}=\exp \left(\left|\left(y_{1}-y_{2}\right)\right|\right)$, where $y_{1}$ and $y_{2}$ are the rapidities of the two jets with the highest $p_{T}$. The choice of this variable is motivated by the fact that in Rutherford scattering the $\chi_{\text {dijet }}$ distribution is independent from $\left|\left(y_{1}-y_{2}\right)\right|$. This feature provides signatures of new physics that have a different angular distribution than given by QCD predictions, and can be determined more readily as they can produce an excess of events at small values of $\chi_{\text {dijet }}$. The measurement of dijet angular distributions, unfolded for detector effects and in bins of dijet mass, is shown in Figure 9. The measured distributions are found to be in agreement with predictions from perturbative QCD that include electroweak corrections.

This search can therefore constrain models of quark contact interactions, extra spatial dimensions, quantum black holes, and dark matter. The exclusion limits for these kind of models are reported in Figure 10.

\section{Dark Matter interpretation}

Three of the searches presented can be interpreted in the same theoretical model. In this model a DM mediator (Z') with unknown mass $M_{M e d}$ is assumed to be a spin-1 particle (vector or axial- 


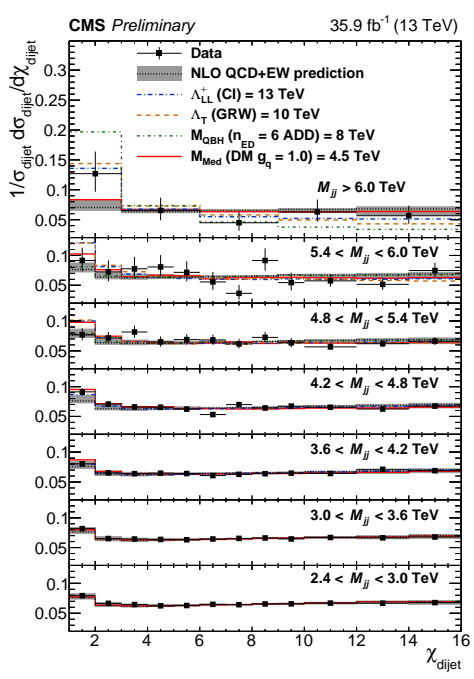

\begin{tabular}{|c|c|c|}
\hline Model & Observed lower limit (TeV) & Expected lower limit (TeV) \\
\hline$\Lambda_{\mathrm{LL} / \mathrm{RR}}^{+}(\mathrm{NLO})$ & 13.1 & $15.2 \pm 0.9$ \\
\hline$\Lambda_{\mathrm{LL} / \mathrm{RR}}^{\mathrm{L} / \mathrm{NLO})}$ & 17.4 & $23.9 \pm 3.0$ \\
\hline$\Lambda_{\mathrm{VV}}^{+}(\mathrm{NLO})$ & 15.1 & $17.3 \pm 1.0$ \\
\hline$\Lambda_{\mathrm{VV}}^{\mathrm{V}}(\mathrm{NLO})$ & 22.2 & $31.2 \pm 3.8$ \\
\hline$\Lambda_{\mathrm{AA}}^{+}(\mathrm{NLO})$ & 15.2 & $17.3 \pm 1.0$ \\
\hline$\Lambda_{\mathrm{AA}}^{\mathrm{AA}}(\mathrm{NLO})$ & 22.1 & $31.0 \pm 3.8$ \\
\hline$\Lambda_{(\mathrm{V}-\mathrm{A})}^{+\mathrm{A}}(\mathrm{NLO})$ & 9.1 & $11.7 \pm 1.0$ \\
\hline$\Lambda_{(\mathrm{V}-\mathrm{A})}^{-}(\mathrm{NLO})$ & 9.3 & $11.9 \pm 1.1$ \\
\hline $\operatorname{ADD} \Lambda_{\mathrm{T}}(\mathrm{GRW})$ & 10.6 & $12.1 \pm 0.9$ \\
\hline $\mathrm{ADD} M_{\mathrm{S}}(\mathrm{HLZ}) n_{E D}=2$ & 11.4 & $13.3 \pm 1.0$ \\
\hline $\mathrm{ADD} M_{\mathrm{S}}(\mathrm{HLZ}) n_{E D}=3$ & 12.6 & $14.4 \pm 1.1$ \\
\hline $\mathrm{ADD} M_{\mathrm{S}}(\mathrm{HLZ}) n_{E D}=4$ & 10.6 & $12.1 \pm 0.9$ \\
\hline $\mathrm{ADD} M_{\mathrm{S}}(\mathrm{HLZ}) n_{E D}=5$ & 9.6 & $10.9 \pm 0.8$ \\
\hline $\mathrm{ADD} M_{\mathrm{S}}(\mathrm{HLZ}) n_{E D}=6$ & 8.9 & $10.2 \pm 0.8$ \\
\hline $\mathrm{QBH} M_{\mathrm{OBH}}\left(\mathrm{ADD} n_{E D}=6\right)$ & 8.3 & $8.7 \pm 0.3$ \\
\hline $\mathrm{QBH} M_{\mathrm{OBH}}\left(\operatorname{RS} n_{E D}=1\right)$ & 6.0 & $6.5 \pm 0.4$ \\
\hline & & \\
\hline
\end{tabular}

Figure 10: Observed and expected exclusion limits at 95\% CL for various benchmark models. [5]

Figure 9: Normalized $\chi_{\text {dijet }}$ distributions shown for the unfolded data (points) compared to NLO predictions (black dotted line) [5].

vector) and to decay only to pairs of quarks and pairs of DM particles, with mass $m_{D M}$, and with a universal quark coupling $g_{q}$ and a DM coupling $g_{D M}$.

Figure 11 shows the excluded values at $95 \% \mathrm{CL}$ of mediator mass as a function of $m_{D M}$ for vector mediators for a choice of couplings $g_{q}=0.25$ and $g_{D M}=1$. It can be noticed that the dijet analysis and the boosted dijet analysis are complementary excluding two different regions in this plane. However these searches are valid only for narrow resonances which means a width $\Gamma \sim 10 \%$ corresponding to a coupling $g_{q} \sim 0.4 \%$. Therefore higher coupling cannot be probed with these analyses. The dijet angular analysis instead allows to exclude higher quark coupling given that is not restricted to narrow resonance. It's clear therefore that these searches are all complementary covering as much as possible the phase-space (mass and width/coupling).

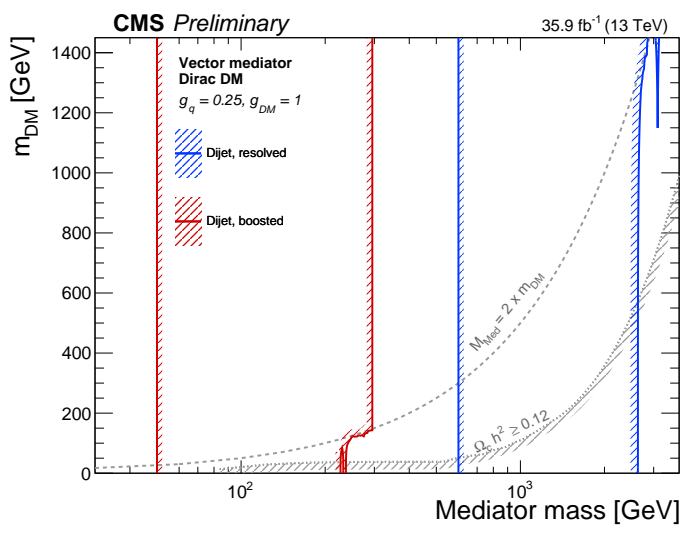

Figure 11: The 95\% CL excluded regions from boosted dijet search (red) in the plane of dark matter mass vs. mediator mass, for vector mediators. The excluded regions from the dijet analysis are shown in blue. The exclusion is computed with coupling choice $g_{q}=0.25$ and $g_{D M}=1$. [3] 


\section{References}

[1] CMS Collaboration, "The CMS experiment at the CERN LHC", JINST 3 S08004 (2008)

[2] CMS Collaboration, CMS Physics Analysis Summary CMS-PAS-EXO-16-056, 2016, http://cds.cern.ch/record/2256873? ln=en

[3] CMS Collaboration, CMS Physics Analysis Summary CMS-PAS-EXO-17-001, 2016, http://cds.cern.ch/record/2264843? ln=en

[4] CMS Collaboration, CMS Physics Analysis Summary CMS-PAS-EXO-16-029, 2016, http://cds.cern.ch/record/2231062?ln=en

[5] CMS Collaboration, CMS Physics Analysis Summary CMS-PAS-EXO-16-046, 2016, http://cds.cern.ch/record/2273455?ln=en 